\title{
SEA-Based Multi-dimensional Space Description Model of Equipment Support Capability
}

\author{
Li Si $^{\mathrm{a}, *}$, Gu Ping ${ }^{\mathrm{c}}$, Sun Wang-hu ${ }^{\mathrm{d}}$ \\ Department of Equipment Command \&Management \\ Ordnance Engineering College \\ Shijiazhuang, PRC \\ e-mail: lisi8569@sina.com.
}

\author{
Ma Shao-chuang \\ Department of Training \\ Ordnance Engineering College \\ Shijiazhuang, PRC \\ e-mail: lisi8569@sina.com.
}

\begin{abstract}
Aiming at the traditional methods of research equipment support capability to the lack of scientific quantitative description of the overall support capability, It builds the multi-dimensional space description Model of equipment support capability based on SEA Initially, introduces the basic idea of the modeling, analysis the equipment support ability of model generating method, focuses on the study of building the basic process and form, and puts forwards the calculation model of the whole description of equipment support ability.
\end{abstract}

Keywords-equipment support capability; description model; multi-dimensional space; mapping

\section{INTRODUCTION}

The basic condition is the accurate description of equipment support capability for building the model and research evolution problem of equipment support capability. At present, the existing method mainly divided into two kinds for the description of the equipment support ability ${ }^{[1]}$ : one is the quantitative description of equipment support capability according to specialty; another is the description the equipment support capability from the whole. The two methods are lack of scientific quantitative description for the overall support capability. Multi-dimensional space description model is mainly integrated application the capability space describe method of SEA (System Effectiveness Analysis), described the elements relationship and evolution trend of equipment support capability is allaround and systemic.

\section{BASIS THINKING OF MODELING}

Through the building the multi-dimensional European space by Dimensions of support capability elements, and research on the indexes of bounded function relation between support needs and support capability, we can find the aggregate of satisfies the support requirements form the space, which is called demand mapping. Through research on the indexes of bounded function relation between system state and support capability, find the aggregate of satisfies the system state form the space, called system mapping, get the system efficiency by analyzing the relationship between demand mapping and system mapping.

\section{SPACE DESCRIPTION METHOD BASED ON THE ABILITY OF SEA}

SEA method is an effective method to research ability of the space, and analysis the relationship through the system space and demand space mapping to the ability of the space system corresponding trajectories and demand the relationship between the system performance metrics ${ }^{[2]}$. The establishment of the main creative work in the ability of space, system mapping and demand the establishment of the three aspects, including ability space for the significance of this study is very big, can be used as army equipment support ability of the main description method, through this description, also can build the bridge between equipment support ability and efficiency of equipment support.

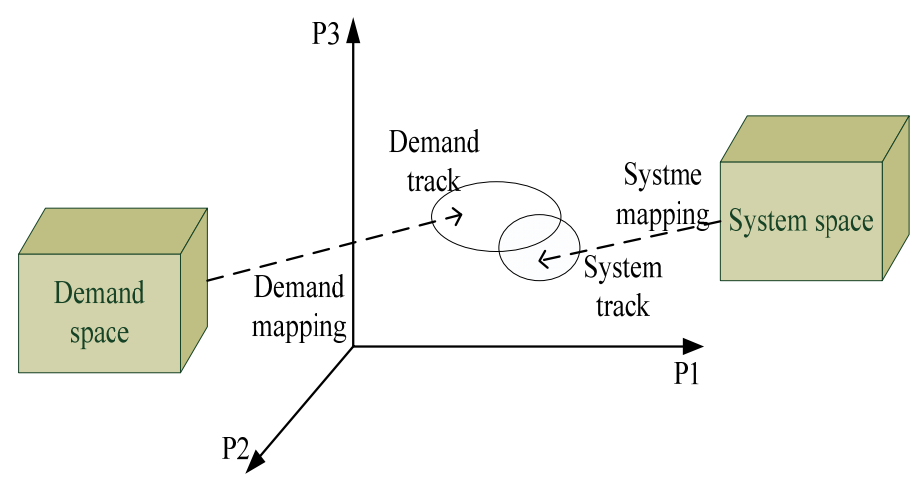

Figure 1. SEA method

SEA method has the clear military meaning and easy to application, and other advantages, the description of equipment support ability is the space vector in the form of equipment support ability, in addition to the support capacity size and direction, the equipment support ability vector and demand mapping contrast, it can be obtained if the task of meeting demand.

\section{EQUIPMENT SUPPORT ABILITY RELATION MODEL GENERATION METHOD}

Army equipment support ability of relational model is a superface of the manifestation of an n-dimension equipment support ability, superface consists of just to meet the 
demands of operational point, superface in the space distribution to describe the elements of the relationship between equipment support ability. Super on each point $\mathrm{P}$ $(\mathrm{X} 1, \mathrm{X} 2, \ldots, \mathrm{Xn})$ (equipment support vector) of each component $\mathrm{X} 1, \mathrm{X} 2, \ldots$, the numerical $\mathrm{Xn}$, is a group to meet the demands of operational equipment support plan.

For the relation model research of the army equipment support ability, we give the following definition:

Demand mapping: it is a task by demand space to the ability of the space mapping; in particular, demand mapping is that changing the original mission parameters of domain requirements into the ability to safeguard domain of a mapping. The mathematical model description is: using function $f_{u}: F \rightarrow u$ represents the bounded function relation between the index and the demand index, its domain for equipment support ability space is P's subset, u represents demand index, it is usually expressed in the probability of security task value, such as the probability of repair equipment.

Ability to satisfy demand tracks: a $p_{l} \in p$, for a given data form uk, $\forall p \in p_{l}, f_{k}(p) \geq w_{k}$ and $p$, is the ability to satisfy demand tracks . In equipment support ability space, meet the needs of a certain operational mapping path form for a specific area, the area can meet the demands of operational superface segmentation that joint surface is called ability needs to meet superface $\mathbf{M}$, for a given data form uk, $\forall p \in \mathbb{M}, f_{k}(p)=w_{\bar{k}}$, and the superface reflects the relationship of support ability.

So, study the demand mapping, obtain ability to satisfy demand track $\mathrm{P}$, and eventually generating capacity requirement to meet superface $M$ process, is the process of establishing equipment support ability factor models.

For single time point equipment support ability, whether to meet the demand of operations is the standard, through the data mining, it gives us the classification model, and its manifestation is a five dimensions (multidimensional) in the space which is a curved surface (superface).

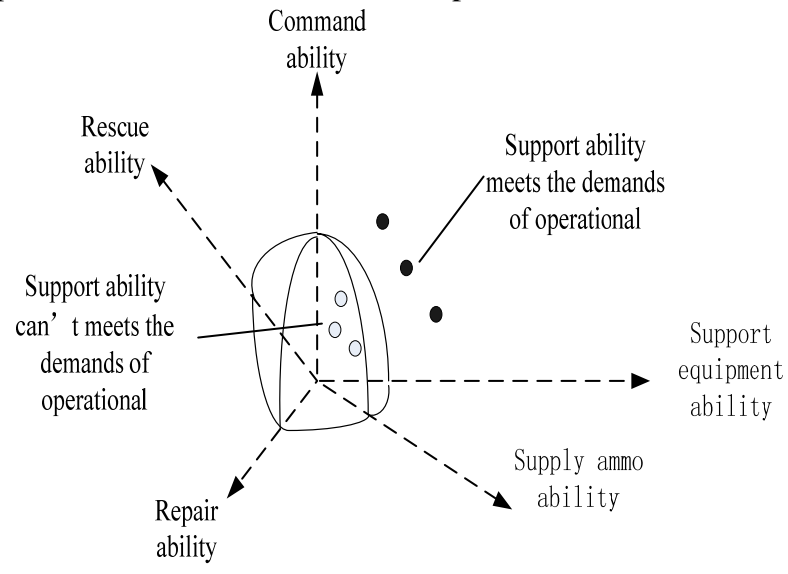

Figure 2. single time point ability to meet the needs of classification model

Equipment support (points) team will work under the integrated joint operations system, to achiver a number of support tasks ${ }^{[3]}$. Different support taskes have different demand of support ability, and the mapping values will be different, the mapping in that form can be divided into five types: ordinary function (can obtained analytical expressions of differential equations (group), unable to get the analytical solutions of differential equations (group), data forms, the simulation experiment and mathematical programming. And the ordinary function is the most ideal form, which can come to the analytical solution, but getting this shows the function of the mission is not much, Usually, we need to use the after four kinds of forms to get the mapping solution, the solving process of the four forms often need to use the numerical approximation method. This paper use s EA (exploratory analysis) method to carry on the analysis, under the support of the condition of simulation experiment, the implementation steps are as follows: first, making a decision goals, it is a safeguard ability value; Secondly, explore in different patterns, different season, under the security demand of the need to guarantee ability the demand of the track, which is the ability to meet demand - M. This part work is under the supporting of the simulation, the key is the numerical approximation method.

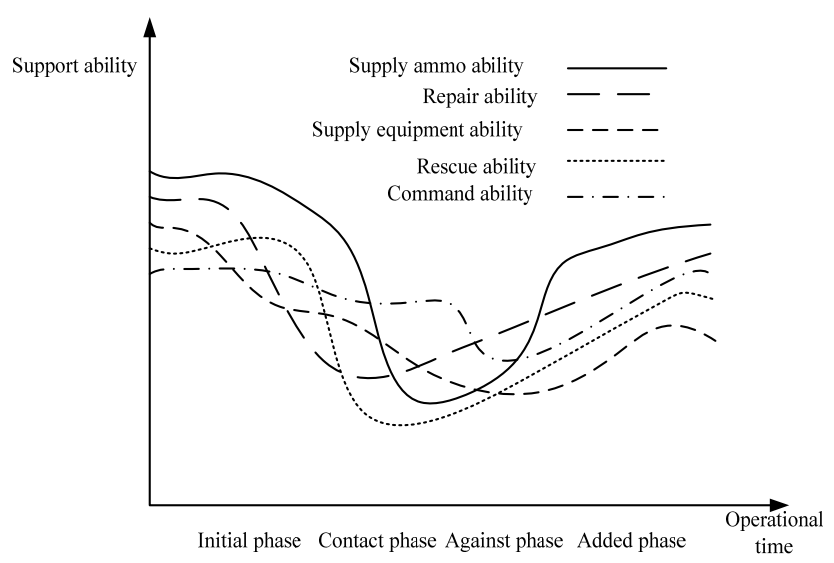

Figure 3. more time forces equipment safeguard the index to the Lord into the direction of multi-dimensional projection curve display method

\section{BULIDING THE EQUIPMENT SUPPORT ABILITY SPACE}

The basic method process of equipment support ability of space construction is: the first, the military experts analysis forces equipment support ability index system qualitative, then using mathematical method to choose, according to the analysis of equipment support needs, establish multidimensional space equipment support ability.

The first is to analysis unit equipment support ability qualitative. The classification analysis method of army equipment support ability index selection is based on its operational needs, the work will be done by military experts.

The second is to determine the key elements, which is to reduce ability index. Its main work is to establish a satisfy the completeness, testability, sensitivity, independence and other requirements of the contracted equipment safeguard index, in order to adapt to the follow-up demand of mathematical analysis. The methods which are used to cut 
capacity index number of main methods are ${ }^{[4]}$ : the use of the comprehensive index; the use of variance analysis and significant factors, number; the use of reduction of principal component analysis index. The work will be done by military personnel and cooperation.

The third is to establish a power space. The main work is to use the reduction indicators as dimensions, constructed an equipment support ability space, such as establishing the equipment rescue ability index space, which can be described by three indexes: quantity, average operation time and the number of rescue resources occupation, in the space of a little said the troops of equipment support ability. But index in variety formes such as the cost model, efficiency model and so on [5], which is also needed to carry on the standardization of processing. Index standardization methods including vector norm method, equivalent coefficient method, comparative method, etc., according to the analysis, we can use it flexibility. In addition, sometimes also need to standard the method such as "bigger is better" type index, "smaller is better", " close to a certain value nearer is the better" and so on , for analysis.

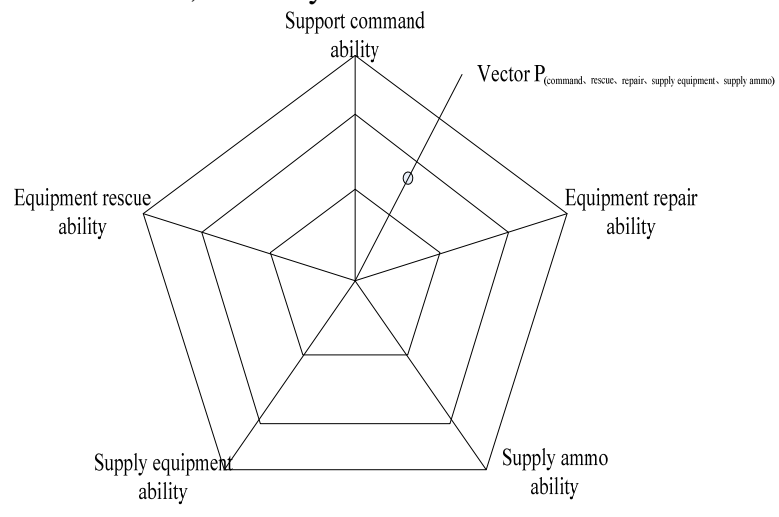

Figure 4. units equipment support ability of multidimensional space description model

To describe the army equipment support ability objectively, we use the filve elements for dimension in the military theory,and the equipment support ability space model is established, any equipment support ability of the form (efficiency) is one of the space vector $P$ $(\mathrm{x} 1, \mathrm{x} 2, \ldots, \mathrm{xn})$. Equipment support ability space: set $\mathrm{Rn}$ as n-dimension Euclidean space, set $\mathrm{P} \subset \mathrm{Rn}, \mathrm{P}=\mathrm{p} 1 \times \mathrm{p} 2 \times \ldots \mathrm{pn}, \mathrm{P}$ is ability index point set, that is $\mathrm{P}(\mathrm{x} 1, \mathrm{x} 2, \ldots, \mathrm{xn})$ (equipment support vector), $\mathrm{X}$ represent capabilities, each dimension represents an ability index.

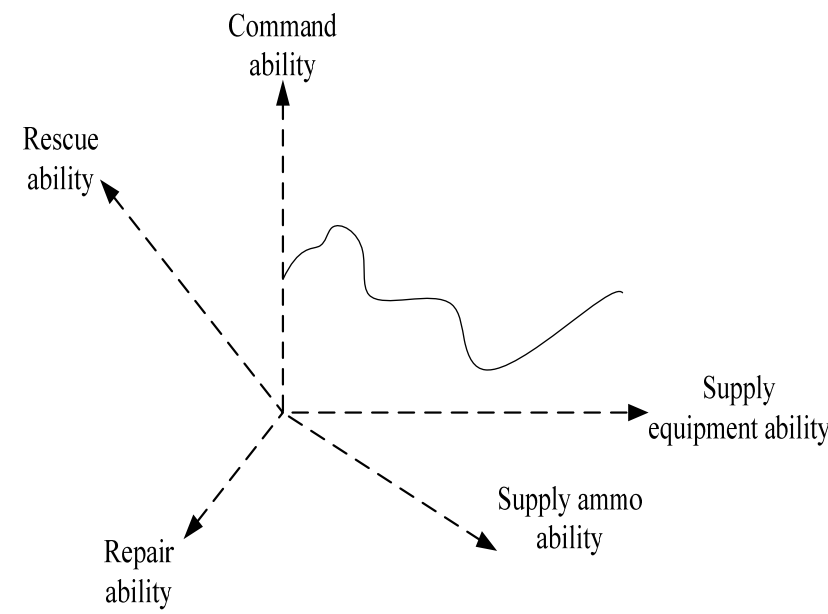

Figure 5. units equipment support ability description model several points in time

To describe the whole process of equipment support ability in space model in equipment support ability objectively, we use a continuous curve to describe the continuous change of the combat ability, after processing, its form is a group of vector $(\mathrm{P} 1, \mathrm{P} 2, \ldots, \mathrm{Pn})$.

\section{THE DESCRIPTION MODEL OF EQUIPMENT SUPPORT ABILITY}

To establish equipment support ability description model, first, we should determine that whether army equipment support ability meets the demand of combat mission that is the ability index validity analysis. When carrying on the analysis, it is necessary to definite demand, using the demand as the effectiveness of the system measurement scale. In practical operation, using the ability to satisfy demand tracks as the standard measure of the ability of the actual system, compare the distribution of the data and track ability needs of ability to satisfy demand tracks, find out the effectiveness of the ability to measure. Analysis model is:

$$
\mathrm{E}=\int_{p l} f(p) d p
$$

In this type, the pl for the ability to satisfy demand track; $f(p)$ is the actual system performance indexes of the power of the joint probability density, which can be obtained by the method of mathematical statistics, it is usually a normal distribution; $\mathrm{E}$ is the actual system capability index whether it meets the requirements of the probability value, which is also the effectiveness of the system capability

$(0 \leq \mathbb{E} \leqslant 1$ ), it represents a system that meets a set of specific task requires a measure of the degree. In the actual process of support process, the equipment support abilities are mainly from two aspects:

The first, from the view of cost point, that is, a vector point from the ability space, so that the equipment support 
performance meets the needs of the army, and the lowest price. The model is:

\section{$\left.\operatorname{Min}\left(\mathrm{C}_{k}, p_{0}, p_{k}\right)\right) \quad \sigma . \tau .: \mathbb{E} \geq \mathbb{R}, \mathbb{E}=\int_{p l} f\left(p, p_{e}\right) d p$}

In this type, $\mathrm{C}\left(p_{0}, p_{e}\right)$ from ability point $p_{0}$ development to $p_{e}$ price;

$f\left(p, p_{\varepsilon}\right)$ is based on vector $p_{\varepsilon}$, to expect the ability index of the joint probability density function;

$\mathrm{K}$ is the demand of equipment support efficiency.

From the Angle of the commander, the second is to pay more attention on the dual problem: how to realize the maximization of security performance with the condition of the limit resources. The model is as follows:

$$
\operatorname{Max}\left(\int_{p} f\left(p, p_{k}\right) d p\right) \text { s.t.: } C\left(p_{b}, p_{k}\right) \leq C_{k}
$$

Type of $G_{\mathbb{R}_{i}}$ is the given resource.

\section{CONCLUSION}

In order to improve the army equipment support ability, the author puts forwards a method to describe the equipment support ability based on SEA. It analyzes the generating method relationship model of the equipment support ability, and constructs the space model of equipment support ability, sets up the description model of equipment support ability. And it gives us a new thought and references to find influence force equipment support tasks of professional ability, find out the key factors influencing the changes of equipment support ability, improve the efficiency of equipment command, guide the construction of army equipment support. But due to the operation of the model lack of passing the test of practice, the deficiencies and weaknesses is not analysis and research in-depth.

\section{REFERENCES}

[1] Michael Boito, Kevin Brancato, John C. The Air Force's Experience with Should-Cost Reviews and Options for Enhancing Its Capability to Conduct Them[R]. RAND, 2012.

[2] Andrew G. Loerch, Larry B. Rainey. Methods for Conducting Military Operational Analysis[M]. Military Operations Research S, 2007.

[3] ZHAI Huai-yu,BAI Yan-qi,SHEN Yao-de,etc.Research on the Structure of Combat Entity Conceptual Model[J].Command Control \& Simulation ,Vol. 33(5) (2011),pp.8-11.

[4] J. Evermann. A UML and OWL description of Bunge's upper-level ontology model [J]. Software and Systems Modeling, Vol. 8(2) (2009),pp.235-249.

[5] HU Peng,SHAO Chen-xi,YANG Ming,etc.Conceptual Model Representation of Military Simulation System Based on Qualitative Feedback[J],Journal of System Simulation, Vol. 20(2) (2008), pp.449-452. 